\title{
Modular Classes of Loday Algebroids
}

\author{
Mathieu Stiénon * \\ E.T.H. Zürich \\ stienon@math.ethz.ch
}

\author{
Ping $\mathrm{Xu}^{\dagger}$ \\ Penn State University \\ ping@math.psu.edu
}

\begin{abstract}
We introduce the concept of Loday algebroids, a generalization of Courant algebroids. We define the naive cohomology and modular class of a Loday algebroid, and we show that the modular class of the double of a Lie bialgebroid vanishes. For Courant algebroids, we describe the relation between the naive and standard cohomologies and we conjecture that they are isomorphic when the Courant algebroid is transitive.
\end{abstract}

\section{Naive Cohomology}

Given a Courant algebroid $(E, \rho, \llbracket \cdot, \cdot \rrbracket,\langle\cdot, \cdot\rangle)$, let $\Gamma\left(\wedge^{k} \operatorname{ker} \rho\right)$ denote the space of smooth sections of the (possibly singular) vector bundle $\wedge^{k} \operatorname{ker} \rho$ (i.e. smooth sections $\alpha$ of $\wedge^{k} E$ such that $\left.\alpha\right|_{m} \in \wedge^{k} \operatorname{ker} \rho$ for each $\left.m \in M\right)$. The extension of the pseudo-metric $\langle\cdot, \cdot\rangle$ to $\wedge^{k} E$ naturally induces an isomorphism $\Xi: \wedge^{k} E \rightarrow \wedge^{k} E^{*}$. Since, by definition, $\langle\mathcal{D} f, e\rangle=\frac{1}{2} \rho(e) f$, the sections of $\wedge^{k} \operatorname{ker} \rho$ are characterized as the elements $\varepsilon \in \Gamma\left(\wedge^{k} E\right)$ such that $\breve{\imath}_{\mathcal{D} f} \varepsilon=0$, $\forall f \in C^{\infty}(M)$. Here $\breve{\imath}_{\mathcal{D} f}=\Xi^{-1}{ }_{\circ} i_{\mathcal{D} f \circ} \Xi$, where $i_{\mathcal{D} f}: \Gamma\left(\wedge^{k+1} E^{*}\right) \rightarrow \Gamma\left(\wedge^{k} E^{*}\right)$ is the usual contraction of exterior forms with the section $\mathcal{D} f \in \Gamma(E)$. Define an operator $\breve{d}: \Gamma\left(\wedge^{k} \operatorname{ker} \rho\right) \rightarrow \Gamma\left(\wedge^{k+1} E\right)$ by

$$
\begin{aligned}
(\breve{d} \alpha)\left(e_{0}, \cdots, e_{k}\right)=\sum_{i=0}^{k}( & -1)^{i} \rho\left(e_{i}\right) \alpha\left(e_{0}, \cdots, \widehat{e_{i}}, \cdots, e_{k}\right) \\
& +\sum_{i<j}(-1)^{i+j} \alpha\left(\llbracket e_{i}, e_{j} \rrbracket, e_{0}, \cdots, \widehat{e_{i}}, \cdots, \widehat{e_{j}}, \cdots, e_{k}\right),
\end{aligned}
$$

for all $\alpha \in \Gamma\left(\wedge^{k} \operatorname{ker} \rho\right)$ and $e_{0}, \ldots, e_{k} \in \Gamma(E)$. Here the pairing between $\Gamma\left(\wedge^{k} \operatorname{ker} \rho\right)$ and $\Gamma\left(\wedge^{k} E\right)$ is via the identification $\Xi: \wedge^{k} E \rightarrow \wedge^{k} E^{*}$. The following Lemma follows from the Courant algebroid properties, in particular the relations $\rho(\mathcal{D} f)=0$ and $\llbracket \mathcal{D} f, e \rrbracket+\mathcal{D}\langle\mathcal{D} f, e\rangle=0$.

${ }^{*}$ Research supported by the European Union through the FP6 Marie Curie R.T.N. ENIGMA (Contract number MRTN-CT-2004-5652).

${ }^{\dagger}$ Research partially supported by NSF grant DMS-0605725 \& NSA grant H98230-06-1-0047. 
Lemma 1.1 We have $\breve{d} \Gamma\left(\wedge^{k} \operatorname{ker} \rho\right) \subset \Gamma\left(\wedge^{k+1} \operatorname{ker} \rho\right)$. Moreover, $\left(\Gamma\left(\wedge^{\bullet} \operatorname{ker} \rho\right), \breve{d}\right)$ is a cochain complex.

The cohomology of this cochain complex is called the naive cohomology of $E$ and is denoted $H_{\text {naive }}(E)$.

Remark 1.2 It is easy to see that a 1-cochain $\theta \in \Gamma(\operatorname{ker} \rho)$ is a 1-cocycle if, and only if, $\langle\theta, \llbracket a, b \rrbracket\rangle=\rho(a)\langle\theta, b\rangle-\rho(b)\langle\theta, a\rangle$ for all $a, b \in \Gamma(E)$, and a 1-coboundary if, and only if, $\theta=\mathcal{D} f$ for some $f \in C^{\infty}(M)$.

Remark 1.3 Let $V$ be the $C^{\infty}(M)$-module generated by $\mathcal{D}\left(C^{\infty}(M)\right)$. Since $\langle\mathcal{D} f, a\rangle=\frac{1}{2} \rho(a) f$, we have $V=\Gamma\left(\operatorname{ker} \rho^{\perp}\right)$ and $\Xi(V)=\Gamma\left(\operatorname{ker} \rho^{0}\right)=\rho^{*}\left(\Gamma\left(T^{*} M\right)\right)$. Moreover $V \subset \Gamma(\operatorname{ker} \rho)$, for $\rho_{\circ} \mathcal{D}=0$. Therefore, when $(E, \rho, \llbracket \cdot, \cdot \rrbracket,\langle\cdot, \cdot\rangle)$ is a regular Courant algebroid (i.e. $\rho$ has constant rank), $E / \Xi^{-1}\left(\rho^{*} T^{*} M\right)$ is a Lie algebroid and $H_{\text {naive }}^{\bullet}(E)$ is the cohomology of this Lie algebroid. However, in general, $\Gamma(E) / V$ is only a Lie-Rinehart algebra over $C^{\infty}(M)$. One can consider $H_{\text {naive }}(E)$ as its cohomology [4].

Example 1.4 When $E=T M \oplus T^{*} M$ is an exact Courant algebroid, $H_{\text {naive }}^{\bullet}(E)$ is isomorphic to the de Rham cohomology of $M$.

Example 1.5 If $E$ is a Courant algebroid over a point, i.e. a Lie algebra equipped with a non-degenerate ad-invariant bilinear form, $H_{\text {naive }}^{\bullet}(E)$ is simply the Lie algebra cohomology.

\section{Relation with standard cohomology}

Courant algebroids can also be obtained as derived brackets [6] 9] using degree two super-symplectic manifolds. More precisely, given a Courant algebroid $(E, \rho, \llbracket \cdot, \cdot \rrbracket,\langle\cdot, \cdot\rangle), E[1]$ is a super-Poisson manifold, where the Poisson structure is induced by the pseudo-metric. There is a minimal symplectic realization $X \stackrel{\pi}{\rightarrow} E[1]$ and a cubic function $\Theta$ on $X$ such that $\{\Theta, \Theta\}=0$ and, for all $f \in C^{\infty}(M)$ and $e_{1}, e_{2} \in \Gamma(E)$,

$$
\mathcal{D} f=\{\Theta, f\} \quad \text { and } \quad e_{1} \circ e_{2}=\left\{\left\{\Theta, e_{1}\right\}, e_{2}\right\},
$$

where the symbol $\circ$ denotes the asymmetric Dorfman bracket defined by the relation $a \circ b=\llbracket a, b \rrbracket+\mathcal{D}\langle a, b\rangle$. Here elements in $\Gamma\left(\wedge^{k} E\right)$ are viewed as functions of degree $k$ on $X$ by considering them as functions on $E[1]$ via the pseudo-metric $\langle\cdot, \cdot\rangle$ and identifying them with their pull back by $\pi$. Similarly functions on $M$ are also identified with their pull back in $X$. By $\mathcal{A}^{k}$ we denote the space of functions on $X$ of degree $k$. Then $\left(\mathcal{A}^{\bullet},\{\Theta, \cdot\}\right)$ is a cochain complex. Its cohomology is called the standard cohomology by Roytenberg [9] and we shall denote it by $H_{\text {std }}^{\bullet}(E)$.

Lemma 2.1 1. If $c \in \Gamma\left(\wedge^{k} \operatorname{ker} \rho\right)$, then $\{\Theta, c\}=\breve{d} c$;

2. If $c \in \Gamma\left(\wedge^{k} E\right)$ satisfies $\{\Theta, c\}=0$, then $c \in \Gamma\left(\wedge^{k} \operatorname{ker} \rho\right)$ and $\breve{d} c=0$. 
Proof. (i) It suffices to prove the case when $k=1$. The general situation follows from the Leibniz rule. Now since $\rho(c)=0$, we have $\forall e_{1}, e_{2} \in \Gamma(E)$,

$$
\begin{aligned}
& \left\langle c \circ e_{2}, e_{1}\right\rangle-(\breve{d} c)\left(e_{1}, e_{2}\right) \\
= & \left(-\left\langle e_{2} \circ c, e_{1}\right\rangle+2\left\langle\mathcal{D}\left\langle c, e_{2}\right\rangle, e_{1}\right\rangle\right)-\left(\rho\left(e_{1}\right)\left\langle c, e_{2}\right\rangle-\rho\left(e_{2}\right)\left\langle c, e_{1}\right\rangle-\left\langle c, \llbracket e_{1}, e_{2} \rrbracket\right\rangle\right) \\
= & \rho\left(e_{2}\right)\left\langle c, e_{1}\right\rangle-\left\langle e_{2} \circ c, e_{1}\right\rangle-\left\langle c, \llbracket e_{2}, e_{1} \rrbracket\right\rangle \\
= & \rho\left(e_{2}\right)\left\langle c, e_{1}\right\rangle-\left\langle e_{2} \circ c, e_{1}\right\rangle-\left\langle c, e_{2} \circ e_{1}\right\rangle \\
= & 0 .
\end{aligned}
$$

It thus follows that $\left\{\left\{\{\Theta, c\}, e_{2}\right\}, e_{1}\right\}-\left\{\left\{\breve{d c}, e_{2}\right\}, e_{1}\right\}=0$, which implies that $\{\Theta, c\}=\breve{d} c$.

(ii) Since $\breve{\iota}_{\mathcal{D} f} c=\{\mathcal{D} f, c\}=\{\{\Theta, f\}, c\}=\{f,\{\Theta, c\}\}=0$ for all $f \in C^{\infty}(M)$, we have $c \in \Gamma\left(\wedge^{k} \operatorname{ker} \rho\right)$.

As a consequence, we have a homomorphism $\phi: H_{\text {naive }}^{\bullet}(E) \rightarrow H_{\text {std }}^{\bullet}(E)$. Lemma 2.1 also implies that $\phi$ is an isomorphism in degrees 0 and 1. It is natural to ask when $\phi$ is an isomorphism in all degrees. When $E$ is a Courant algebroid over a point, $\phi$ is clearly an isomorphism. On the other hand, when $E$ is the standard Courant algebroid $T M \oplus T^{*} M$, both $H_{\text {naive }}^{\bullet}(E)$ and $H_{\text {std }}^{\bullet}(E)$ are isomorphic to the de Rham cohomology of $M$. Hence $\phi$ is also an isomorphism. This leads to the following

Conjecture When $E$ is a transitive Courant algebroid, $\phi$ is an isomorphism.

\section{Lie derivatives and Loday algebroids}

The Lie derivative of Courant algebroids was introduced in [10. Let us recall its definition briefly. An infinitesimal automorphism of the vector bundle $E \stackrel{\pi}{\rightarrow} M$ is a vector field on $E$ - a derivation of the algebra $C^{\infty}(E)$ - which preserves the subspaces $\pi^{*} C^{\infty}(M)$ and $\Gamma(E)$ (whose elements are identified with functions linear on the fibers of $\pi$ through the pairing $\langle\cdot, \cdot\rangle)$. In other words, it is a covariant differential operator on $E$, i.e. a pair of differential operators $\delta^{0}$ : $C^{\infty}(M) \rightarrow C^{\infty}(M)$ and $\delta^{1}: \Gamma(E) \rightarrow \Gamma(E)$ satisfying

$$
\delta^{0}(f g)=f \delta^{0}(g)+\delta^{0}(f) g \quad \text { and } \quad \delta^{1}(f e)=f \delta^{1}(e)+\delta^{0}(f) e,
$$

for any $f, g \in C^{\infty}(M)$ and $e \in \Gamma(E)$. It is known [9] that the Lie algebra aut $(E)$ of infinitesimal automorphisms of the Courant algebroid $E$ consists of those covariant differential operators $\delta=\left(\delta^{0}, \delta^{1}\right)$ on $E$ which satisfy the additional properties:

$\delta^{0}\left\langle e_{1}, e_{2}\right\rangle=\left\langle\delta^{1} e_{1}, e_{2}\right\rangle+\left\langle e_{1}, \delta^{1} e_{2}\right\rangle \quad$ and $\quad \delta^{1} \llbracket e_{1}, e_{2} \rrbracket=\llbracket \delta^{1} e_{1}, e_{2} \rrbracket+\llbracket e_{1}, \delta^{1} e_{2} \rrbracket$,

for all $e_{1}, e_{2} \in \Gamma(E)$. 
For any $e \in \Gamma(E)$, the pair $\delta_{e}=\left(\delta_{e}^{0}, \delta_{e}^{1}\right)$ defined by the relations $\delta_{e}^{0}(f)=$ $\rho(e) f$ and $\delta_{e}^{1}(x)=e \circ x$ is an infinitesimal automorphism of the Courant algebroid $E$, i.e. $\delta_{e} \in \mathfrak{a u t}(E)$. Let us denote the (local) flow generated by the vector field on $E$ corresponding to $\delta_{e}$ by $\phi_{t}$. By abuse of notations, we use the same symbol $\phi_{t}\left(\right.$ resp. $\left.\phi_{t}^{*}\right)$ to denote its induced flow on the tensor bundles $E_{j}^{i}=\left(\otimes^{i} E\right) \otimes$ $\left(\otimes^{j} E^{*}\right)(i, j \in\{0,1,2, \ldots\})$ (resp. the induced action on the spaces of sections of the $E_{j}^{i}$ 's). For any section $\sigma \in \Gamma\left(E_{j}^{i}\right)$, define the Lie derivative $\mathcal{L}_{z} \sigma \in \Gamma\left(E_{j}^{i}\right)$ by $\mathcal{L}_{z} \sigma=\left.\frac{d}{d \tau} \phi_{\tau}^{*} \sigma\right|_{\tau=0}$. Thus we have the usual identity: $\left.\frac{d}{d \tau} \phi_{\tau}^{*} \sigma\right|_{\tau=t}=\phi_{t}^{*}\left(\mathcal{L}_{z} \sigma\right)$. In the following proposition, we give a list of important properties of this Lie derivative.

Proposition 3.1 For all $f, g \in C^{\infty}(M)$ and $x, y, z \in \Gamma(E)$, we have:

$$
\begin{gathered}
\mathcal{L}_{z} f=\rho(z) f \quad \mathcal{L}_{z} x=z \circ x \quad \mathcal{L}_{\mathcal{D} f} x=0 \quad \mathcal{L}_{x} \mathcal{D} f=\mathcal{D} \mathcal{L}_{x} f, \\
{\left[\delta, \mathcal{L}_{z}\right]=\mathcal{L}_{\delta^{1} z} \quad \forall \delta \in \mathfrak{a u t}(E),} \\
\mathcal{L}_{z}(\sigma \otimes \tau)=\mathcal{L}_{z} \sigma \otimes \tau+\sigma \otimes \mathcal{L}_{z} \tau \quad \forall \sigma, \tau \in \oplus_{i, j} E_{j}^{i}, \\
\mathcal{L}_{\llbracket x, y \rrbracket}=\left[\mathcal{L}_{x}, \mathcal{L}_{y}\right], \\
\mathcal{L}_{z} \llbracket x, y \rrbracket=\llbracket \mathcal{L}_{z} x, y \rrbracket+\llbracket x, \mathcal{L}_{z} y \rrbracket, \\
\mathcal{L}_{f x} y=f \mathcal{L}_{x} y-(\rho(y) f) x+2\langle x, y\rangle \mathcal{D} f, \\
\mathcal{L}_{z}\langle x, y\rangle=\left\langle\mathcal{L}_{z} x, y\right\rangle+\left\langle x, \mathcal{L}_{z} y\right\rangle .
\end{gathered}
$$

Definition 3.2 A Loday algebroid consists of a vector bundle $\pi: E \rightarrow M, a$ pseudo-metric $\langle\cdot, \cdot\rangle$ on the fibers of $\pi$, a bundle map $\rho: E \rightarrow T M$ and an $\mathbb{R}$-bilinear operation $\circ$ on $\Gamma(E)$ satisfying

$$
\begin{gathered}
e_{1} \circ\left(e_{2} \circ e_{3}\right)=\left(e_{1} \circ e_{2}\right) \circ e_{3}+e_{2} \circ\left(e_{1} \circ e_{3}\right), \\
\rho\left(e_{1} \circ e_{2}\right)=\left[\rho\left(e_{1}\right), \rho\left(e_{2}\right)\right], \\
e_{1} \circ\left(f e_{2}\right)=\left(\rho\left(e_{1}\right) f\right) e_{2}+f\left(e_{1} \circ e_{2}\right), \\
e_{1} \circ e_{2}+e_{2} \circ e_{1}=2 \mathcal{D}\left\langle e_{1}, e_{2}\right\rangle, \\
\mathcal{D} f \circ e=0,
\end{gathered}
$$

where $\mathcal{D}: C^{\infty}(M) \rightarrow \Gamma(E)$ is the $\mathbb{R}$-linear map defined by $\langle\mathcal{D} f, e\rangle=\frac{1}{2} \rho(e) f$.

Remark 3.3 1. According to [11], for Courant algebroids, axioms (91) and (10) are redudant. It would be interesting to investigate if it is also the case for Loday algebroids.

2. The Leibniz algebroids studied by several authors [3, [2, 12] are a more general notion.

3. A Courant algebroid is a Loday algebroid satisfying the additional axiom $\rho(e)\left\langle e_{1}, e_{2}\right\rangle=\left\langle e \circ e_{1}, e_{2}\right\rangle+\left\langle e_{1}, e \circ e_{2}\right\rangle$.

Lemma 3.4 If $(E, \rho, \circ,\langle\cdot, \cdot\rangle)$ is a Loday algebroid, then $\rho(\mathcal{D} f)=0$ and $\llbracket \mathcal{D} f, e \rrbracket+$ $\mathcal{D}\langle\mathcal{D} f, e\rangle=0$, for all $f \in C^{\infty}(M)$ and $e \in \Gamma(E)$. Here $\llbracket x, y \rrbracket=\frac{1}{2}(x \circ y-y \circ x)$ as in a Courant algebroid. 
Proof. Applying $\rho$ to both sides of (11) and making use of (9), we get $\rho\left(\mathcal{D}\left\langle e_{1}, e_{2}\right\rangle\right)=0$ for any $e_{1}, e_{2} \in \Gamma(E)$ and thus also $\rho\left(\mathcal{D}\left\langle f e_{1}, e_{2}\right\rangle\right)=0$ for any $f \in C^{\infty}(M)$. The Leibniz rule $\mathcal{D}(f g)=g \mathcal{D} f+f \mathcal{D} g$ implies that $\rho(\mathcal{D} f)=0$. The other relation follows immediately from (12) and (11).

As a consequence, the definition of the naive cohomology extends from Courant algebroids to Loday algebroids.

Let $(E, \rho, \circ,\langle\cdot, \cdot\rangle)$ be a Loday algebroid. Given a section $z \in \Gamma(E)$, set $\mathcal{L}_{z} f=\rho(z) f$ for $f \in C^{\infty}(M)$ and $\mathcal{L}_{z} x=z \circ x$ for $x \in \Gamma(E)$ and extend $\mathcal{L}_{z}$ to $\Gamma\left(\wedge^{k} E\right)$ by the Leibniz rule.

Proposition 3.5 Identities (4), (5) and (6) still hold for any Loday algebroid.

Remark 3.6 It is unknown if the standard cohomology can be defined for Loday algebroids. Indeed, it would be interesting to see if there exists a derived bracket in the sense of Kosmann-Schwarzbach [6] for a Loday algebroid.

\section{Modular classes}

A Loday algebroid module is a vector bundle $S \rightarrow M$ endowed with an $\mathbb{R}$-linear map $\Gamma(E) \otimes \Gamma(S) \rightarrow \Gamma(S): e \otimes s \mapsto \nabla_{e} s$ satisfying

$$
\begin{array}{ll}
\nabla_{\mathcal{D} f} s=0 & \nabla_{e}(f s)=f \nabla_{e} s+(\rho(e) f) s \\
\nabla_{f e} s=f \nabla_{e} s & \nabla_{e_{1}}\left(\nabla_{e_{2}} s\right)-\nabla_{e_{2}}\left(\nabla_{e_{1}} s\right)=\nabla_{\llbracket e_{1}, e_{2} \rrbracket} s
\end{array}
$$

for any $f \in C^{\infty}(M), e, e_{1}, e_{2} \in \Gamma(E)$ and $s \in \Gamma(S)$.

Now let $S$ be a real line bundle which is a module of the Loday algebroid $E$. Assume that there exists a nowhere zero section $s \in \Gamma(S)$. The relation $D_{e} s=$ $\left\langle\theta_{s}, e\right\rangle s$ defines a section $\theta_{s} \in \Gamma(E)$. From $\nabla_{\mathcal{D} f} s=0$, it follows that $\rho\left(\theta_{s}\right)=0$. And from (14), it follows that $\theta_{s}$ is a naive 1-cocycle. Finally, (13) implies that, for any nowhere vanishing function $f \in C^{\infty}(M), \theta_{f s}=f \theta_{s}+2 \mathcal{D}(\ln |f|)$. Thus the class $\left[\theta_{s}\right] \in H_{\text {naive }}^{1}(E)$ is independent of the chosen section $s$ and only depends on the module $S$. We will denote this class by $\theta_{S}$. As in [1, 7, when the line bundle $S$ is not trivial, we set $\theta_{S}=\frac{1}{2} \theta_{S \otimes S}$, where $S \otimes S$ is necessarily a trivial real line bundle. We call $\theta_{S}$ the modular class of the module $S$.

Theorem 4.1 Given a Loday algebroid $(E, \rho, \circ,\langle\cdot, \cdot\rangle), \wedge^{\text {top } E}$ is an E-module with $\nabla=\mathcal{L}$.

Proof. It remains to prove that $\mathcal{L}_{f e} s=f \mathcal{L}_{e} s$ for any $f \in C^{\infty}(M)$ and $s \in$ $\Gamma\left(\wedge^{t o p} E\right)$. According to Proposition 3.1, for any $f \in C^{\infty}(M)$ and $e, a \in \Gamma(E)$, we have

$$
\begin{aligned}
\mathcal{L}_{f e} a=f \mathcal{L}_{e} a-(\rho(a) f) e+2\langle e, a\rangle \mathcal{D} f & =f \mathcal{L}_{e} a-2\langle\mathcal{D} f, a\rangle e+2\langle e, a\rangle \mathcal{D} f \\
& =\left(f \mathcal{L}_{e}-2(e \wedge) \circ \breve{\iota}_{\mathcal{D} f}+2(\mathcal{D} f \wedge) \circ \breve{\iota}_{e}\right)(a)
\end{aligned}
$$


Note that, as differential operators on $\Gamma(\wedge \bullet E), \mathcal{L}_{f e}, f \mathcal{L}_{e}, 2(e \wedge) \circ \breve{\mathcal{L}}_{\mathcal{D} f}$ and $2(D f \wedge) \breve{\iota}_{e}$ all are derivations of degree 0 with respect to the wedge product on $\Gamma(\wedge \bullet E)$. Since $\mathcal{L}_{f e}$ and $\left.f \mathcal{L}_{e}-2(e \wedge) \circ \breve{\mathcal{D}}_{\mathcal{D} f}+2(D f \wedge)\right)_{\circ} \breve{\imath}_{e}$ are equal when acting both on sections of $E$ and on functions on $M$, they are also equal when extended to $\Gamma\left(\wedge^{*} E\right)$. In particular, if $s \in \Gamma\left(\wedge^{t o p} E\right)$,

$$
\mathcal{L}_{f e} s=f \mathcal{L}_{e} s-2(e \wedge) \circ \breve{\mathcal{D}}_{\mathcal{D} f} s+2(\mathcal{D} f \wedge) \circ \breve{\imath}_{e} s=f \mathcal{L}_{e} s-2\langle e, \mathcal{D} f\rangle s+2\langle\mathcal{D} f, e\rangle s=f \mathcal{L}_{e} s
$$

The modular class $\left[\theta_{\wedge^{t o p} E}\right] \in H_{\text {naive }}^{1}(E)$ of the $E$-module $\wedge^{t o p} E$ is called the modular class of the Loday algebroid $E$.

\section{$5 \quad$ Examples}

Let $E=A \oplus A^{*}$ be the double of a Lie bialgebroid $\left(A, A^{*}\right)$ 5. In this case, $\mathcal{D}=\frac{1}{2}\left(d+d_{*}\right)$ and, for all $X, Y \in \Gamma(A)$ and $\xi, \eta \in \Gamma\left(A^{*}\right)$, the bracket on $\Gamma(E)$ is defined by

$$
\begin{gathered}
\llbracket X, \xi \rrbracket=\left(-L_{\xi} X+\frac{1}{2} d_{*}(\xi, X)\right)+\left(L_{X} \xi-\frac{1}{2} d(\xi, X)\right), \\
\llbracket X, Y \rrbracket=[X, Y], \quad \llbracket \xi, \eta \rrbracket=[\xi, \eta] .
\end{gathered}
$$

Now $\wedge^{t o p} E \cong\left(\wedge^{t o p} A\right) \otimes\left(\wedge^{t o p} A^{*}\right)$ is a trival line bundle. For the sake of simplicity, we assume that there exists a nowhere vanishing section $V \in \Gamma\left(\wedge^{\text {top }} A\right)$. Let $\Omega \in \Gamma\left(\wedge^{t o p} A^{*}\right)$ be its dual section. For any $X \in \Gamma(A)$ and $\xi \in \Gamma\left(A^{*}\right)$, one has $\mathcal{L}_{X} \xi=\llbracket X, \xi \rrbracket+\mathcal{D}\langle X, \xi\rangle=-L_{\xi} X+d_{*}(\xi, X)+L_{X} \xi=-i_{\xi} d_{*} X+L_{X} \xi$. It follows from the Leibniz rule (see Proposition 3.1) that, for any $\sigma \in \Gamma\left(\wedge^{k} A^{*}\right)$, $\mathcal{L}_{X} \sigma=L_{X} \sigma+\lambda$ where $\lambda \in \Gamma\left(A \otimes\left(\wedge^{k-1} A^{*}\right)\right)$. On the other hand, since $A$ is isotropic with respect to $\langle\cdot, \cdot\rangle$, we have that $\mathcal{L}_{X} \tau=L_{X} \tau$ if $\tau \in \Gamma\left(\wedge^{k} A\right)$. It thus follows that

$$
\mathcal{L}_{X}(V \wedge \Omega)=\left(\mathcal{L}_{X} V\right) \wedge \Omega+V \wedge\left(\mathcal{L}_{X} \Omega\right)=\left(L_{X} V\right) \wedge \Omega+V \wedge\left(L_{X} \Omega\right)=0 .
$$

Similarly we have $\mathcal{L}_{\xi}(V \wedge \Omega)=0$, for all $\xi \in \Gamma\left(A^{*}\right)$. Thus we have proved

Theorem 5.1 If a Courant algebroid is the double of a Lie bialgebroid, then its modular class vanishes.

Example 5.2 If $E=T M \oplus T^{*} M$ is an exact Courant algebroid, the Courant bracket is given by

$$
\llbracket X+\xi, Y+\eta \rrbracket=[X, Y]+i_{X \wedge Y} \phi+L_{X} \eta-L_{Y} \xi+\frac{1}{2} d((\xi, Y)-(\eta, X)),
$$

for all $X, Y \in \mathfrak{X}(M)$ and $\xi, \eta \in \Omega^{1}(M)$. Here $\phi$ is a closed 3-form.

Take a nowhere zero $V \in \Gamma\left(\wedge^{\text {top }} T M\right)$ and its dual $\Omega \in \Omega^{\text {top }}(M)$. One easily sees that $\mathcal{L}_{X} V=L_{X} V+V^{\prime}$, where $V^{\prime} \in \Gamma\left(T^{*} M \otimes \wedge^{\text {top-1 }} T M\right)$ and $\mathcal{L}_{X} \Omega=L_{X} \Omega$. Thus it follows that $\mathcal{L}_{X}(V \wedge \Omega)=L_{X}(V \wedge \Omega)=0, \forall X \in \Gamma(T M)$. One also sees that $\mathcal{L}_{\xi}(V \wedge \Omega)=0, \forall \xi \in \Gamma\left(T^{*} M\right)$. Therefore the modular class vanishes. 
Acknowledgments We thank Zhang-Ju Liu, Alan Weinstein and the referee for useful comments.

\section{References}

[1] Evens, S., Lu, J.-H., and Weinstein, A., Transverse measures, the modular class, and a cohomology pairing for Lie algebroids, Quart. J. Math. Oxford (2) 50 (1999), 417-436.

[2] Grabowski, J. and Urbański, P., Algebroids - general differential calculi on vector bundles. J. Geom. Phys. 31 (1999), no. 2-3, 111-141.

[3] Ibanez, R., de Leon, M., Marrero, J. C., and Padrón, E., Leibniz algebroid associated with a Nambu-Poisson structure, J. Phys. A 32 (1999), 81298144 .

[4] Huebschmann, J., Duality for Lie-Rinehart algebras and the modular class, J. Reine Angew. Math. 510 (1999), 103-159.

[5] Liu, Z.-J., Weinstein, A. and Xu, P., Manin triples for Lie bialgebroids, J. Differential Geom. 45 (1997), 547-574.

[6] Kosmann-Schwarzbach, Y., From Poisson algebras to Gerstenhaber algebras, Ann. Inst. Fourier 46 (1996), 1243-1274.

[7] Kosmann-Schwarzbach, Y. and Weinstein, A., Relative modular classes of Lie algebroids C. R. Math. Acad. Sci. Paris 341 (2005) 8, 509-514.

[8] Loday, J.-L., Une version non commutative des algèbres de Lie: les algèbres de Leibniz, Enseign. Math. 39 (1993), 269-293.

[9] Roytenberg, D., On the structure of graded symplectic supermanifolds and Courant algebroids, Quantization, Poisson brackets and beyond (Manchester, 2001), 169-185, Contemp. Math. 315 Amer. Math. Soc., Providence, RI, 2002.

[10] Stiénon, M., Moser lemma in generalized complex geometry, arXiv:math/0702718.

[11] Uchino, K., Remarks on the Definition of a Courant Algebroid, Letters in Math. Phys. 60 (2002), 171-175.

[12] Wade, A., On some properties of Leibniz algebroids, Infinite dimensional Lie groups in geometry and representation theory (Washington, DC, 2000), 65-78, World Sci. Publ., River Edge, NJ, 2002. 